\title{
Resultados da artroplastia total de disco cervical com acompanhamento mínimo de 10 anos*
}

\section{Results of Total Cervical Disc Replacement with a Minimum Follow-up of 10 Years}

\author{
João Pedro Ferraz Montenegro Lobo ${ }^{1}$ Daniela Vilas Boas Rosa Linhares ${ }^{1}$ José Miguel Monteiro Lopes ${ }^{1}$ \\ Pedro Cacho Rodrigues ${ }^{1}$ Rui Peixoto Pinto ${ }^{1}$ Nuno Silva Morais Neves $2,3,4$ \\ ${ }^{1}$ Departamento de Ortopedia, Hospital São João, Porto, Portugal \\ 2 Serviço de Ortopedia, Centro Hospitalar de São João, Porto, Portugal \\ ${ }^{3}$ Departamento de Cirurgia e Fisiologia, Faculdade de Medicina, \\ Endereço para Correspondência João Pedro Ferraz Montenegro Lobo, \\ MD, Department of Orthopedics of Centro Hospitalar São João \\ Universidade do Porto, Porto, Portugal \\ ${ }^{4}$ Instituto de Investigação i Inovação em Saúde (i3S), Universidade do \\ Hospital, Avenida Prof. Hernâni Monteiro, 4202-451 Porto, Portugal \\ (e-mail: joao_2523@hotmail.com).
}

Porto, Porto, Portugal

Rev Bras Ortop 2020;55(2):185-190.

\section{Resumo}

\section{Palavras-chave}

- substituição total de disco

- artroplastia

- vértebra cervical
Objetivo No presente estudo, apresentamos os resultados com um acompanhamento mínimo de 10 anos da artroplastia total do disco cervical (ATDC) em um nível. Métodos Estudo retrospectivo de pacientes submetidos a ATDC em um nível. Os resultados clínicos incluíram o índice de incapacidade relacionada ao pescoço (IIRP) e a escala visual analógica (EVA) no período pré-operatório, um ano pós-operatório e um mínimo de 10 anos de acompanhamento. Os parâmetros radiográficos incluíram a mobilidade cervical, lordose segmentar, ângulo C2-C7, ossificação heterotópica $(\mathrm{OH})$, degeneração facetária e articular (DFA) e doença do segmento adjacente (DSA).

Resultados Identificados 22 pacientes, 16 mulheres e 6 homens com média de idade de 39,7 anos (26-51 anos), dos quais 15 tiveram um acompanhamento mínimo de 10 anos. Foi verificada melhoria estatisticamente significativa do IIRP e EVA $(p<0,001)$ entre préoperatório e pós-operatório. ( 1 ano ou $>10$ anos). Ao final de 10 anos, $\mathrm{OH}$ foi observada em $59 \%$ dos casos. A mobilidade do implante foi preservada em $80 \%$ dos pacientes. Houve evidência radiológica de DSA em 6 pacientes (40\%). Não houve correlação entre os parâmetros clínicos avaliados e a presença de DSA ou as diferentes classes de $\mathrm{OH}$.

Conclusão Melhoria clínica em todos os parâmetros avaliados, que persiste ao longo do tempo. A maioria dos implantes manteve a mobilidade, como já demonstrado em estudos anteriores com acompanhamentos mais curtos. Numa percentagem significativa, a DSA estava presente, questionando o conceito da tecnologia de preservação de movimento. No entanto, sem nenhuma intervenção cirúrgica por esse motivo, uma vez que não houve correlação com piores resultados clínicos.
Objective In the present study, we present the results with at least 10 years of followup of the cervical disc prosthesis implanted in a single level.

Estudo desenvolvido no Departamento de Ortopedia, Hospital São João, Porto, Portugal.

recebido

29 de Junho de 2018

aceito

22 de Janeiro de 2019
DOI https://doi.org/

10.1055/s-0039-3400526. ISSN 0102-3616.
Copyright $\odot 2020$ by Sociedade Brasileira License terms de Ortopedia e Traumatologia. Published by Thieme Revinter Publicações Ltda, Rio de Janeiro, Brazil 


\author{
Keywords \\ - total disc \\ replacement \\ - arthroplasty \\ - cervical vertebrae
}

\begin{abstract}
Methods Retrospective study of patients undergoing single-level total cervical disc replacement (TCDR). Clinical results included the neck disability index (NDI) and the visual analogue scale (VAS) in the preoperative period, one year postoperatively, and a minimum of 10 years of follow-up. The radiographic parameters included cervical mobility, segmental lordosis, $\mathrm{C2}-\mathrm{C} 7$ angle, heterotopic ossification ( $\mathrm{HO})$, facet and joint degeneration (FJD) and adjacent segment disease (ASD).

Results We identified 22 patients, 16 women and 6 men with mean age of 39.7 years old (26-51 years old), of which fifteen completed a minimum follow-up of 10 years. There was a statistically significant improvement of NDI and VAS $(p<0.001)$ between the preoperative and the postoperative periods ( 1 year or $>10$ years). At the end of 10 years, $\mathrm{HO}$ was observed in $59 \%$ of the cases. The mobility of the implant was preserved in $80 \%$ of the patients. Radiological evidence of ASD was recorded in 6 patients (40\%). There was no correlation between the clinical parameters evaluated and the presence of ASD or the different classes of HO.

Conclusion Clinical improvement in all evaluated parameters, which persists over time. Most implants maintained mobility, as has already been demonstrated in other studies with shorter follow-ups. In a significant percentage of cases, ASD was observed, questioning the concept of motion preserving technology. However, we did not have any surgical intervention for this reason, since there was no correlation with worse clinical results.
\end{abstract}

\section{Introdução}

A artroplastia total do disco cervical (ATDC) é um procedimento cirúrgico alternativo à artrodese. Em teoria, mantendo o movimento no nível operado, a artroplastia pode diminuir a demanda por níveis adjacentes, subsequente degeneração e doença do segmento adjacente (DSA). Vários estudos randomizados demonstraram a equivalência ou até a superioridade da ATDC em termos de resolução da dor e/ou sucesso neurológico em comparação com discectomia cervical anterior e fusão intersomática. ${ }^{1-4}$ No entanto, sua indicação, benefício clínico, custos, segurança e complicações em longo prazo permanecem incertos.

No presente estudo, apresentamos os resultados com pelo menos 10 anos de acompanhamento da prótese de disco cervical implantada em um único nível.

\section{Materiais e Métodos}

Estudo retrospectivo de pacientes submetidos a ATDC (Prestige ou Bryan, Medtronic, Dublin, Irlanda) de um único nível, de acordo com o critério de inclusão ( - Tabela 1 ), entre janeiro de 2003 e dezembro de 2006.

Tabela 1 Critérios de inclusão para o estudo

\begin{tabular}{|l|}
\hline Critérios de inclusão \\
\hline $\begin{array}{l}\text { Doença degenerativa sintomática do disco cervical em um nível com } \\
\text { cervicalgia e/ou braquialgia e/ou déficits neurológicos confirmados por } \\
\text { ressonância magnética }\end{array}$ \\
\hline Cirurgia em um nível cervical e entre C5-C7 \\
\hline Idade entre 18 e 65 anos durante o período da cirurgia \\
\hline Maus resultados com tratamento conservador \\
\hline Consentimento informado do paciente \\
\hline
\end{tabular}

Resultados clínicos incluíram o índice de incapacidade relacionada ao pescoço (IIRP) e a escala visual analógica (EVA) em relação à gravidade e frequência da dor cervical e braquialgia avaliadas no pré-operatório, após um ano de pósoperatório e depois de um mínimo de 10 anos de acompanhamento. Também foi analisada a perspectiva do paciente em relação ao sucesso da cirurgia, complicações e subsequentes intervenções cirúrgicas.

Todas as avaliações radiográficas foram realizadas por um observador independente. Foram obtidas as radiografias anteroposteriores (APs), lateral e dinâmica (flexão/extensão). Os parâmetros radiográficos incluíram mobilidade cervical $\left(\geq 2^{\circ}\right.$ de variação nas visualizações dinâmicas), lordose segmentar, ângulo $\mathrm{C2}-\mathrm{C} 7$, ossificação heterotópica $(\mathrm{OH})$ de acordo com McAfee et $\mathrm{al}^{5}{ }^{5}$ osteólise, degeneração facetária e articular (DFA), migração e translação. A presença e gravidade de DSA também foi avaliada.

Os critérios para a presença de DSA foram:

- 1-Degeneração do disco e/ou DFA (ambas de grau 0 a 4 de acordo com Côté et $\mathrm{al}^{6}$ ) $\geq$ grau 2 e mínimo de 1 grau maior que no pré-operatório;

- 2-Nova instabilidade ( $\geq 3,5 \mathrm{~mm}$ de translação e/ou $\geq 11^{\circ} \mathrm{de}$ variação rotacional em relação aos segmentos adjacentes);

- 3-Instrumentação adicionada.

As cirurgias foram realizadas por diferentes cirurgiões em 2 hospitais. Os exames de acompanhamento foram realizados por investigadores ou outros cirurgiões em seus respectivos hospitais. Estudo aprovado pelo comitê de ética em saúde.

\section{Resultados}

Identificamos 22 pacientes, 16 mulheres e 6 homens com idade média de 39,7 anos (26-51 anos). Oito pacientes possuíam 
próteses não restritivas (Bryan ) e 14 possuíam próteses semirrestritivas (Prestige). Quinze completaram um acompanhamento mínimo de 10 anos. Todos os pacientes apresentavam cervicobraquialgia (68,75\% no lado esquerdo e $31,25 \%$ no lado direito). Em 68,75\% dos pacientes, o nível operado foi C5/C6, e em $31,25 \%$ o nível operado foi C6/C7. Houve uma melhora estatisticamente significativa do IIRPe da $\operatorname{EVA}(p<0,001)$ entre o pré-operatório e o pós-operatório (1 ano). Uma melhora estatisticamente significativa na EVA foi observada após pelo menos 10 anos de acompanhamento em comparação com 1 ano de pós-operatório $(p<0,001)$. O IIRP permaneceu estável ao final de 10 anos $(p=0,63)$ ( - Tabela 2 ).

Um total de $75 \%$ dos pacientes estava muito satisfeito/satisfeito, e 94\% repetiriam e recomendavam o procedimento. Ao final de 10 anos, $\mathrm{OH}$ foi observada em $59 \%$ dos casos, sendo $41 \%$ de grau $0,26 \%$ de grau $1,13 \%$ de grau 3, e $20 \%$ de grau 4 . A mobilidade do implante foi preservada em $80 \%$ dos pacientes. A lordose cervical préoperatória $\left(\sim 6,3^{\circ}\right)$ aumentou significativamente no período pós-operatório imediato $\left(\sim 13,5^{\circ}\right)$, mas reduziu-se significativamente entre essa avaliação e o pós-operatório mínimo de 10 anos $\left(\sim 9,8^{\circ}\right)$. A lordose segmentar aumentou significativamente entre o pré-operatório $\left(\sim 4,1^{\circ}\right)$ e o pós-operatório inicial $\left(\sim 5,8^{\circ}\right)$, mas diminuiu no acompanhamento tardio $\left(\sim 4,9^{\circ}\right)$. Evidências radiológicas de DSA foram registradas em 6 pacientes (40\%) (-Tabela 3). Não houve correlação entre os parâmetros clínicos avaliados e a presença de DSA ou as diferentes classes de $\mathrm{OH}$ durante o acompanhamento. Um paciente necessitou de conversão para artrodese cervical anterior no pós-operatório imediato por compressão medular, com déficits neurológicos progressivos.

\section{Discussão}

No presente estudo, observamos que a ATDC apresenta excelentes resultados clínicos que permanecem estáveis por pelo menos 10 anos. Ao considerar uma melhoria significativa no IIRP como uma diferença mínima de 15 pontos, ela foi alcançada em $\sim 87 \%$ ao final do acompanhamento de um ano, e, em seguida, permaneceu estável ao final de pelo menos 10 anos de acompanhamento. Esses resultados estão de acordo com estudos anteriores. Sasso et al $^{7}$ demonstraram uma melhora no IIRP em $90,6 \%$ após um acompanhamento de 4 anos com implante de disco do tipo Bryan. Burkus et $\mathrm{al}^{8}$ observaram uma melhora no IIRP em $83,4 \%$ após um acompanhamento de 7 anos com implante do disco do tipo Prestige.

A ATDC é realizada para manter a mobilidade no nível operado e impedir a hipermobilidade dos níveis adjacentes e consequente aumento do estresse regional. A funcionalidade a longo prazo da ATDC é de particular importância. De acordo com Heller et al, ${ }^{9}$ os resultados de 2 anos de acompanhamento indicaram que a ATDC poderia preservar a mobilidade local e era uma alternativa viável à discectomia cervical anterior com fusão em pacientes com doença persistente sintomática do disco cervical em um nível. 0 mesmo resultado foi observado por Du et al. ${ }^{10}$ Os resultados do nosso estudo indicam que o implante permanece funcional a longo prazo, com mais de $80 \%$ das próteses com mobilidade acima do limiar de $2^{\circ}$ após 10 anos de acompanhamento.

Discectomia cervical anterior com fusão é considerado o padrão na abordagem terapêutica na doença degenerativa do disco cervical com radiculopatia cervical e/ou mielopatia em pacientes que não responderam ao tratamento conservador. ${ }^{11}$ Embora a degeneração em nível adjacente seja observada após a fusão na coluna cervical em 7 a 17\% dos casos, permanece a discussão sobre se esse fenômeno é induzido ou acelerado pela fusão ou se é simplesmente a progressão natural da doença. ${ }^{12-14}$ Alguns estudos demonstraram que de 5,7 a 37\% dos pacientes submetidos à fusão cervical necessitam de nova intervenção cirúrgica para aliviar os sintomas clínicos de DSA com acompanhamento de 3,5 anos. ${ }^{15}$ Embora não haja dados consistentes de que a preservação do movimento do disco impeça ou reduza a ocorrência de degeneração do segmento adjacente, há pelo menos um benefício teórico, especialmente na doença multinível. Por outro lado, a presença de alterações radiográficas não está diretamente associada à doença sintomática. Os casos de intervenção cirúrgica devido à degeneração sintomática do segmento adjacente após a ATDC são escassos até o momento, como demonstrado no presente estudo, apesar da taxa de $40 \%$ de sinais radiológicos de DSA.

A OH depois da ATDC é tema de controvérsia, de uma forma que contraria a preservação do movimento. O mecanismo do desenvolvimento de $\mathrm{OH}$ na coluna cervical ainda não foi claramente definido, mas as possíveis causas parecem ser múltiplas, incluindo doença degenerativa pré-operatória, técnica cirúrgica, design do implante, movimento limitado ou fatores genéticos. ${ }^{16}$ Yi et al $^{17}$ realizaram um estudo com 170 pacientes submetidos a ATDC e encontraram diferenças significativas nas taxas de ocorrência de $\mathrm{OH}$ com base no gênero e tipo de prótese do paciente. Leung et $\mathrm{al}^{18}$ descreveram a incidência e o resultado de $\mathrm{OH}$ em pacientes tratados com o disco cervical Bryan. Um total de $18 \%$ dos 90 pacientes tratados tinham $\mathrm{OH}$, e $6,7 \%$ possuíam graus avançados ( $3^{\circ}$ e $4^{\circ}$ graus) de OH após 1 ano de acompanhamento. $O$ mecanismo detalhado de fisiopatologia e prevenção de $\mathrm{OH}$ requer estudos mais precisos. Após o procedimento de ATDC, a perda de mobilidade devido a OH é uma das possíveis consequências. No entanto, os resultados do presente estudo indicam que a ocorrência de $\mathrm{OH}$ pode não ser uma complicação importante no que diz respeito à sua consequência clínica. Após 10 anos de acompanhamento, três pacientes apresentaram $\mathrm{OH}$ grau 4 sem nenhum movimento do disco intervencionado. Nenhum paciente necessitou de intervenção devido a $\mathrm{OH}$.

Em termos de complicações, relatamos uma complicação grave rara na qual ocorreu compressão neurológica imediata e houve a necessidade de conversão precoce em artrodese anterior. A identificação precoce e o tratamento adequado e imediato dessas possíveis complicações são essenciais para obter um bom resultado nesses pacientes.

O presente estudo tem algumas limitações. Enfatizamos a natureza retrospectiva do estudo. Segundo, ele é composto por uma amostra relativamente pequena. Terceiro, o estudo não possui um grupo de controle. Para resultados e conclusões mais válidos, outros estudos prospectivos rigorosamente planejados deverão ser realizados. 


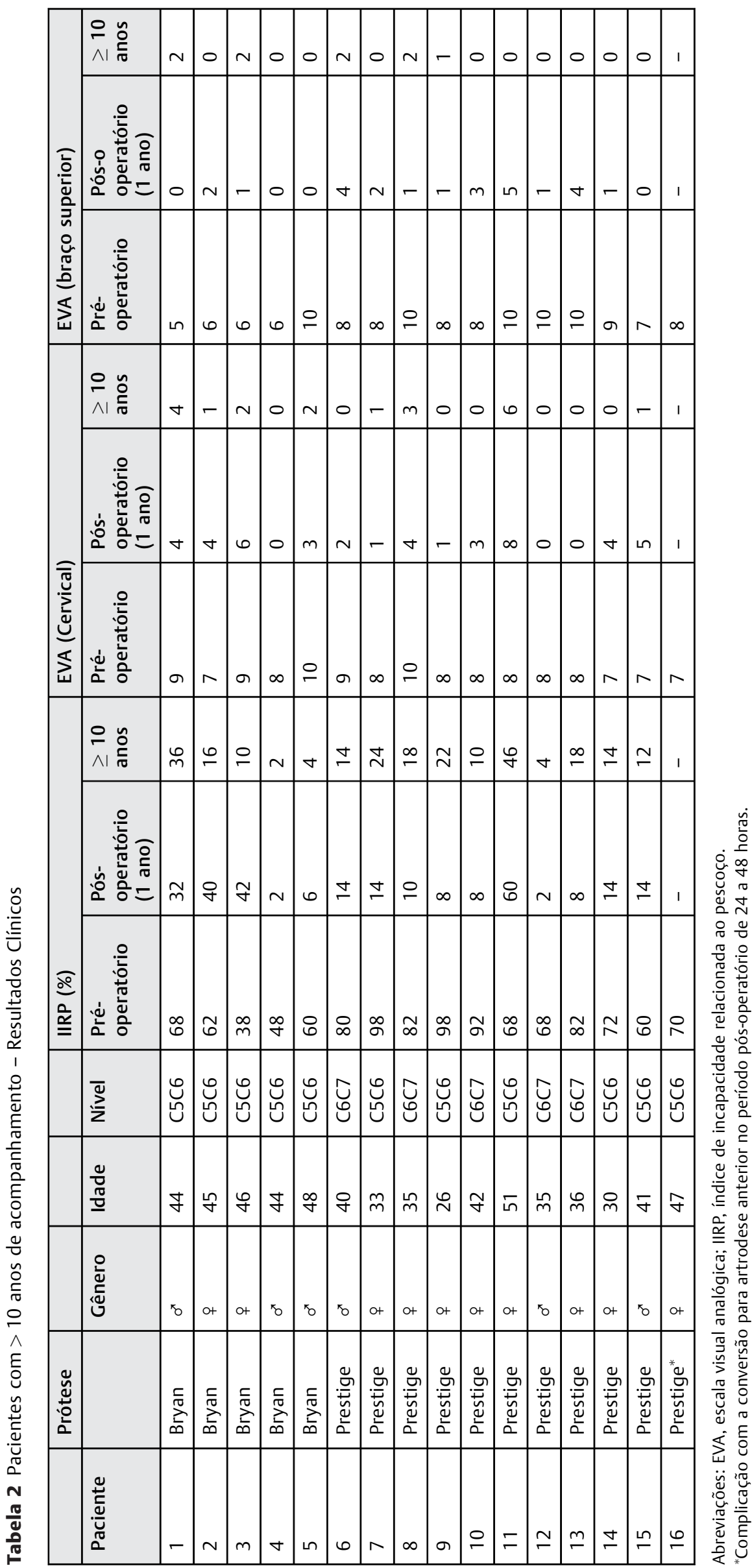


Tabela 3 Pacientes com > 10 anos de acompanhamento - Resultados radiográficos

\begin{tabular}{|c|c|c|c|c|c|c|c|c|c|c|c|c|}
\hline \multirow[b]{2}{*}{ Paciente } & \multicolumn{5}{|c|}{ McAffee } & \multicolumn{3}{|l|}{ Lordose cervical } & \multicolumn{3}{|c|}{ Lordose segmentar } & \multirow[t]{2}{*}{ DSA } \\
\hline & 0 & 1 & II & III & IV & Pré-operatório & $\begin{array}{l}\text { Pós-operatório } \\
\text { (1 ano) }\end{array}$ & $\begin{array}{l}\geq 10 \\
\text { anos }\end{array}$ & Pré-operatório & $\begin{array}{l}\text { Pós-operatório } \\
\text { (1 ano) }\end{array}$ & $\begin{array}{l}\geq 10 \\
\text { anos }\end{array}$ & \\
\hline 1 & $x$ & & & & & 15 & 23 & 15 & 3 & 12 & 3,7 & $x$ \\
\hline 2 & $x$ & & & & & 10 & 11,0 & 10,0 & 4 & 3 & 6,0 & $x$ \\
\hline 3 & $x$ & & & & & 8 & 13,0 & 12 & 3 & 3,2 & 3,2 & $x$ \\
\hline 4 & & & & & $x$ & 14 & 13,0 & 11 & 12 & 13 & 10 & $\sqrt{ }$ \\
\hline 5 & & $x$ & & & & 5 & 10,0 & 7 & 4 & 9 & 5 & $x$ \\
\hline 6 & & & & & $x$ & 9 & 22,0 & 10 & 3 & 3 & 3 & $x$ \\
\hline 7 & $x$ & & & & & 2 & 20,0 & 12 & 2,1 & 2 & 2 & $x$ \\
\hline 8 & $x$ & & & & & 20 & 15,0 & 13,1 & 11 & 10 & 10 & $\sqrt{ }$ \\
\hline 9 & & $x$ & & & & 12 & 25,0 & 18 & 3 & 5 & 5 & $x$ \\
\hline 10 & & $x$ & & & & 10 & 18,0 & 12 & 5 & 3 & 3 & $x$ \\
\hline 11 & $x$ & & & & & 10 & 19,0 & 15 & 0 & 5 & 5 & $\sqrt{ }$ \\
\hline 12 & & & & $x$ & & 9 & 17,0 & 17 & 4 & 7,7 & 6 & $x$ \\
\hline 13 & & & & $x$ & & -17 & $-5,0$ & -5 & 6 & 9 & 9 & $\sqrt{ }$ \\
\hline 14 & & $x$ & & & & -20 & $-9,0$ & -9 & 2 & 2 & 2 & $\sqrt{ }$ \\
\hline 15 & & & & & $x$ & 8 & 10,0 & 9,3 & 0 & 0 & 0 & $\sqrt{ }$ \\
\hline $16^{*}$ & & & & & & - & - & - & - & - & - & - \\
\hline
\end{tabular}

Abreviação: DSA, doença do segmento adjacente.

*Complicação com a conversão para artrodese anterior no período pós-operatório de 24 a 48 horas.

\section{Conclusão}

O presente estudo demonstra bons resultados da ATDC em um nível, com acompanhamento mínimo de 10 anos. Clinicamente, observamos uma melhora em todos os parâmetros avaliados, que persistem ao longo do tempo. Radiologicamente, a maioria dos implantes mantinha a mobilidade. Em uma porcentagem significativa de casos, a DSA foi observada, questionando o conceito e os benefícios da tecnologia de preservação de movimento. No entanto, não registramos nenhuma intervenção cirúrgica por esse motivo, pois não houve correlação com piores resultados clínicos.

Esta técnica não está isenta de complicações e uma seleção rigorosa de pacientes é essencial para obter os melhores resultados.

\section{Conflito de Interesses}

Os autores declaram não haver conflito de interesses.

\section{Referências}

1 Murrey D, Janssen M, Delamarter R, et al. Results of the prospective, randomized, controlled multicenter Food and Drug Administration investigational device exemption study of the ProDisc$C$ total disc replacement versus anterior discectomy and fusion for the treatment of 1-level symptomatic cervical disc disease. Spine J 2009;9(04):275-286

2 Zigler JE, Delamarter R, Murrey D, Spivak J, Janssen M. ProDisc-C and anterior cervical discectomy and fusion as surgical treatment for single-level cervical symptomatic degenerative disc disease: five-year results of a Food and Drug Administration study. Spine 2013;38(03):203-209
3 Hisey MS, Bae HW, Davis R, et al. Multicenter, prospective, randomized, controlled investigational device exemption study comparing mobi-C cervical artificial disc to anterior fusion in the treatment of symptomatic cervical degenerative disc disease. Int J Spine Surg 2014

4 Hisey MS, Bae HW, Davis RJ, et al. Prospective, randomized comparison of cervical total disk replacement versus anterior cervical fusion: Results at 48 Months Follow-up. J Spinal Disord Tech 2015;28(04):E237-E243

5 McAfee PC, Cunningham BW, Devine J, Williams E, Yu-Yahiro J. Classification of heterotopic ossification (HO) in artificial disk replacement. J Spinal Disord Tech 2003;16(04): 384-389

6 Côté P, Cassidy JD, Yong-Hing K, Sibley J, Loewy J. Apophysial joint degeneration, disc degeneration, and sagittal curve of the cervical spine. Can they be measured reliably on radiographs? Spine 1997; 22(08):859-864

7 Sasso RC, Anderson PA, Riew KD, Heller JG. Results of cervical arthroplasty compared with anterior discectomy and fusion: four-year clinical outcomes in a prospective, randomized controlled trial. J Bone Joint Surg Am 2011;93(18):1684-1692

8 Burkus JK, Haid RW, Traynelis VC, Mummaneni PV. Long-term clinical and radiographic outcomes of cervical disc replacement with the Prestige disc: results from a prospective randomized controlled clinical trial. J Neurosurg Spine 2010;13(03): 308-318

9 Heller JG, Sasso RC, Papadopoulos SM, et al. Comparison of BRYAN cervical disc arthroplasty with anterior cervical decompression and fusion: clinical and radiographic results of a randomized, controlled, clinical trial. Spine 2009;34(02):101-107

10 Du J, Li M, Liu H, Meng H, He Q Luo Z. Early follow-up outcomes after treatment of degenerative disc disease with the discover cervical disc prosthesis. Spine J 2011;11(04):281-289

11 Korinth MC. Treatment of cervical degenerative disc disease - current status and trends. Zentralbl Neurochir 2008;69(03):113-124 
190 Resultados da Artroplastia Total de Disco Cervical Lobo et al.

12 Helgeson MD, Bevevino AJ, Hilibrand AS. Update on the evidence for adjacent segment degeneration and disease. Spine J 2013;13 (03):342-351

13 Hilibrand AS, Robbins M. Adjacent segment degeneration and adjacent segment disease: the consequences of spinal fusion? Spine J 2004;4(6, Suppl)190S-194S

14 Ishihara H, Kanamori M, Kawaguchi Y, Nakamura H, Kimura T. Adjacent segment disease after anterior cervical interbody fusion. Spine J 2004;4(06):624-628

15 Murrey DB, Janssen ME, Odum SM, Gottlieb JR, Spector LR, Darden BV. Two-year results of a randomized controlled clinical trial comparing ProDisc-C and Anterior Cervical Discectomy and Fusion. SAS J 2008;2(02):76-85

16 Yang H, Lu X, Yuan W, Wang X, Chen D, Zhao D. Artificial disk replacement in the treatment of degenerative cervical disk disorder: a 30-year follow-up study. Spine 2014;39(19):1564-1571

17 Yi S, Shin DA, Kim KN, et al. The predisposing factors for the heterotopic ossification after cervical artificial disc replacement. Spine J 2013;13(09):1048-1054

18 Leung C, Casey AT, Goffin J, et al. Clinical significance of heterotopic ossification in cervical disc replacement: a prospective multicenter clinical trial. Neurosurgery 2005;57(04):759-763, discussion 759-763 